\title{
Current Status of X-ray Powder Diffraction in Pharmaceutical Industry
}

\author{
Rajni M. Bhardwaj, \\ Small Molecule Design and Development, Eli Lilly \& Company, Indianapolis, IN 46285, USA \\ bhardwaj_rajni@lilly.com
}

Experimental screening is often used to explore the range of possible solid forms (polymorphs, hydrate/ solvates, salts, co-crystals, amorphous) of pharmaceuticals. X-ray powder diffraction (XRPD) is utilized as a primary characterization tool during solid form discovery and drug development cycle to ensure reproducibility of the selected form. Also XRPD data is used to determine the crystal structure of samples where suitable single crystals could not be obtained, using global optimization approaches. However, there are instances where XRPD could not be indexed or crystal structures could not be solved due to presence of phase impurities or high Z' value or higher degree of freedom. For these cases, an alternative approach where crystal structure prediction (CSP) could be used in obtaining crystal structures will be discussed. An example where state-of-the-art analytical tools and recently developed CSP methods were used to determine the crystal structure of every experimentally observed polymorph will be presented. 